\title{
COMPUTATIONAL INTELLIGENCE AND ITS APPLICATIONS
}

Evolutionary Computation, Fuzzy Logic, Neural Network and Support Vector Machine Techniques 
This page intentionally left blank 


\section{COMPUTATIONAL INTELLIGENCE ANDITS APPLICATIONS}

Evolutionary Computation, Fuzzy Logic, Neural Network and Support Vector Machine Techniques

Editors

H. K. Lam

King's College London, UK

S. H. Ling • H. T. Nguyen

University of Technology, Australia 
Published by

Imperial College Press

57 Shelton Street

Covent Garden

London WC2H 9HE

\section{Distributed by}

World Scientific Publishing Co. Pte. Ltd.

5 Toh Tuck Link, Singapore 596224

USA office: 27 Warren Street, Suite 401-402, Hackensack, NJ 07601

UK office: 57 Shelton Street, Covent Garden, London WC2H 9HE

\section{British Library Cataloguing-in-Publication Data}

A catalogue record for this book is available from the British Library.

\section{COMPUTATIONAL INTELLIGENCE AND ITS APPLICATIONS \\ Evolutionary Computation, Fuzzy Logic, Neural Network and Support Vector Machine Techniques}

Copyright $(9) 2012$ by Imperial College Press

All rights reserved. This book, or parts thereof, may not be reproduced in any form or by any means, electronic or mechanical, including photocopying, recording or any information storage and retrieval system now known or to be invented, without written permission from the Publisher.

For photocopying of material in this volume, please pay a copying fee through the Copyright Clearance Center, Inc., 222 Rosewood Drive, Danvers, MA 01923, USA. In this case permission to photocopy is not required from the publisher.

ISBN-13 978-1-84816-691-2

ISBN-10 1-84816-691-5

Printed in Singapore. 


\section{Preface}

Computational intelligence techniques are fast-growing and promising research topics that have drawn a great deal of attention from researchers for many years. This volume brings together many different aspects of the current research on intelligence technologies such as neural networks, support vector machines, fuzzy logics, evolutionary computing and swarm intelligence. The combination of these techniques provides an effective treatment toward some industrial and biomedical applications. Most real-world problems are complex and even ill-defined. Lack of knowledge on the problems or too much information makes the classical analytical methodologies difficult to apply and obtain reasonable results. Computational intelligence techniques demonstrate superior learning and generalization abilities on handling these complex and ill-defined problems. By using appropriate computational intelligence techniques, some essential characteristics and important information can be extracted to deal with the problems. It has been shown that various computational intelligence techniques have been successfully applied to a wide range of applications from pattern recognition and system modeling to intelligent control problems and biomedical applications.

This edited volume provides the state-of-the-art research on significant topics in the field of computational intelligence. It presents fundamental concepts and essential analysis of various computational techniques to offer a systematic and effective tool for better treatment of different applications. Simulation and experimental results are included to illustrate the design procedure and the effectiveness of the approaches. With collective experiences and the knowledge of leading researchers, the important problems and difficulties are fully addressed, concepts are fully explained and methodologies are provided to handle various problems.

This edited volume comprises 13 chapters which falls into 4 main categories: (1) Evolutionary computation and its applications, (2) Fuzzy 
logics and their applications, (3) Neural networks and their applications and (4) Support vector machines and their applications.

Chapter 1 compares three machine learning methods, support vector machines, AdaBoost and soft margin AdaBoost algorithms, to solve the pose estimation problem. Experiment results show that both the support vector machines-based method and soft margin AdaBoost-based method are able to reliably classify frontal and pose images better than the original AdaBoost-based method.

Chapter 2 proposes a particle swarm optimization for polynomial modeling in a dynamic environment. The performance of the proposed particle swarm optimization is evaluated by polynomial modeling based on a set of dynamic benchmark functions. Results show that the proposed particle swarm optimization can find significantly better polynomial models than genetic programming.

Chapter 3 deals with the problem of restoration of color-quantized images. A restoration algorithm based on particle swarm optimization with multi-wavelet mutation is proposed to handle the problem. Simulation results show that it can improve the quality of a half-toned color-quantized image remarkably in terms of both signal-to-noise ratio improvement and convergence rate and the subjective quality of the restored images can also be improved.

Chapter 4 deals with a non-invasive hypoglycemia detection for Type 1 diabetes mellitus (T1DM) patients based on the physiological parameters of the electrocardiogram signals. An evolved fuzzy inference model is developed for classification of hypoglycemia with rule optimization and membership functions using a hybrid particle swarm optimization method with wavelet mutation.

Chapter 5 studies the limit cycle behavior of weights of perceptron. It is proposed that the perceptron exhibiting the limit cycle behavior can be employed for solving a recognition problem when downsampled sets of bounded training feature vectors are linearly separable. Numerical computer simulation results show that the perceptron exhibiting the limit cycle behavior can achieve a better recognition performance compared to a multi-layer perceptron.

Chapter 6 presents an alternative information theoretic criterion (minimum description length) to determine the optimal architecture of neural networks according to the equilibrium between the model parameters and model errors. The proposed method is applied for modeling of various data using neural networks for verification. 
Chapter 7 solves eigen-problems of matrices using neural networks. Several recurrent neural network models are proposed and each model is expressed as an individual differential equation, with its analytic solution being obtained. The convergence properties of the neural network models are fully discussed based on the solutions to these differential equations and the computing steps are designed toward solving the eigen-problems, with numerical simulations being provided to evaluate each model's effectiveness.

Chapter 8 considers the study of methods of automation for the insertion of self-tapping screws. A new methodology for monitoring the insertion of self-tapping screws is developed based on radial basis function neural networks, which are able to generalize and to correctly classify unseen insertion signals. The ability of the artificial neural networks to classify signals belongs to a single insertion case. Both the computer simulation and experimental results show that after a modest training period, the neural network is able to correctly classify torque signature signals.

Chapter 9 applies the theory behind both support vector classification and regression to deal with real-world problems. A classifier is developed which can accurately estimate the risk of developing heart disease simply from the signal derived from a finger-based pulse oximeter. The regression example shows how support vector machines can be used to rapidly and effectively recognize hand-written characters particularly designed for the so-called graffiti character set.

Chapter 10 proposes a control oriented modeling approach to depict nonlinear behavior of heart rate response at both the onset and offset of treadmill exercise to accurately regulate cardiovascular response to exercise for the individual exerciser.

Chapter 11 explores control methodologies to handle time variant behavior for heart rate dynamics at onset and offset of exercises. The effectiveness of the proposed modeling and control approach is shown from the regulation of dynamical heart rate response to exercise through simulation using Matlab.

Chapter 12 investigates real-time fault detection and isolation for heating, ventilation and air conditioning systems by using an online support vector machine. Simulation studies are given to show the effectiveness of the proposed online fault detection and isolation approach.

This edited volume covers state-of-the-art computational intelligence techniques and the materials are suitable for post-graduate students and researchers as a reference in engineering and science. Particularly, it is more suitable for researchers working on computational intelligence 
including evolutionary computation, fuzzy logics, neural networks and support vector machines. Moreover, a wide range of applications using computational intelligence techniques, such as biomedical problems, control systems, forecasting, optimization problems, pattern recognition and system modeling, are covered. These problems can be commonly found in industrial engineering applications. So, this edited volume can be a good reference, providing concept, techniques, methodologies and analysis, for industrial engineers applying computational intelligence to deal with engineering problems.

We would like to thank all the authors for their contributions to this edited volume. Thanks also to the staff members of the Division of Engineering, King's College London and the Faculty of Engineering and Information Technology, University of Technology, Sydney for their comments and support. The editor, H.K. Lam, would like to thank his wife, Esther Wing See Chan, for her patience, understanding, support and encouragement that make this work possible. Last but not least, we would like to thank the publisher Imperial College Press for the publication of this edited volume and the staff who have offered support during the preparation of the manuscript.

The work described in this book was substantially supported by grants from King's College London and University of Technology, Sydney.

H.K. Lam

S.H. Ling

H.T. Nguyen 


\section{Contents}

Preface $\quad$ v

Evolutionary Computation and its Applications 1

1. Maximal Margin Algorithms for Pose Estimation 3 Ying Guo and Jiaming Li

2. Polynomial Modeling in a Dynamic Environment based on a Particle Swarm Optimization

Kit Yan Chan and Tharam S. Dillon

3. Restoration of Half-toned Color-quantized Images Using Particle Swarm Optimization with Multi-wavelet Mutation

Frank H.F. Leung, Benny C.W. Yeung and Y.H. Chan

Fuzzy Logics and their Applications

4. Hypoglycemia Detection for Insulin-dependent Diabetes Mellitus: Evolved Fuzzy Inference System Approach

S.H. Ling, P.P. San and H.T. Nguyen

Neural Networks and their Applications

5. Study of Limit Cycle Behavior of Weights of Perceptron C.Y.F. Ho and B.W.K. Ling 
6. Artificial Neural Network Modeling with Application to Nonlinear Dynamics

Yi Zhao

7. Solving Eigen-problems of Matrices by Neural Networks

Yiguang Liu, Zhisheng You, Bingbing Liu and Jiliu Zhou

8. Automated Screw Insertion Monitoring Using Neural Networks: A Computational Intelligence Approach to Assembly in Manufacturing 183 Bruno Lara, Lakmal D. Seneviratne and Kaspar Althoefer

\section{Support Vector Machines and their Applications}

9. On the Applications of Heart Disease Risk Classification and Hand-written Character Recognition using Support Vector Machines

S.R. Alty, H.K. Lam and J. Prada

10. Nonlinear Modeling Using Support Vector Machine for Heart Rate Response to Exercise

Weidong Chen, Steven W. Su, Yi Zhang, Ying Guo, Nghir Nguyen, Branko G. Celler and Hung T. Nguyen

11. Machine Learning-based Nonlinear Model Predictive Control for Heart Rate Response to Exercise

Yi Zhang, Steven W. Su, Branko G. Celler and Hung T. Nguyen

12. Intelligent Fault Detection and Isolation of HVAC System Based on Online Support Vector Machine

Davood Dehestani, Ying Guo, Sai Ho Ling, Steven W. Su and Hung T. Nguyen 